\title{
Digital Watermarking and Tree Structure Based Image Quality Estimation
}

\author{
Sha Wang \\ School of Information \\ Technology and Engineering, \\ University of Ottawa, \\ 800 King Edward Ave.,Ottawa, \\ Ontario, Canada, K1N 6N5 \\ shawang@site.uottawa.ca
}

\author{
Dong Zheng \\ Advanced Video Systems, \\ Communication Research \\ Centre Canada, \\ 3701 Carling Ave.,Ottawa, \\ Ontario, Canada, K2H 8S2 \\ dong.zheng@crc.ca
}

\author{
Jiying Zhao \\ School of Information \\ Technology and Engineering, \\ University of Ottawa, \\ 800 King Edward Ave.,Ottawa, \\ Ontario, Canada, K1N 6N5 \\ jyzhao@site.uottawa.ca
}

\begin{abstract}
Wa James Tam
Advanced Video Systems, Communication Research Centre Canada, 3701 Carling Ave.,Ottawa, Ontario, Canada, K2H 8S2 james.tam@crc.ca
\end{abstract}

Filippo Speranza

Advanced Video Systems,

Communication Research Centre Canada,

3701 Carling Ave.,Ottawa,

Ontario, Canada, $\mathrm{K} 2 \mathrm{H}$ 8S2

filippo.speranza@crc.ca

\begin{abstract}
A new semi-fragile-watermarking-based quality estimation scheme is proposed in this paper. The watermark is embedded into the tree structure of an image with proper embedding strength decided by statistically analyzing the characteristics of the image. In the watermark embedding, the bit-plane coding mechanism is used to simulate the general distortion that images will undergo. A universal "Ideal Mapping Curve" is generated using the SPIHT (Set Partitioning in Hierarchical Trees) algorithm with different BPP (Bits Per Pixel) settings. In the watermark extraction, the quality of the watermarked images will be estimated under different distortions, such as JPEG compression and Gaussian noise pollution. The "Ideal Mapping Curve" used for quality estimation against different distortions can be achieved by weighting the universal "Ideal Mapping Curve" using the empirical weighting factors. The experimental results suggest that the proposed algorithm works effectively and efficiently.
\end{abstract}

\section{Categories and Subject Descriptors}

I.4.m [Image Processing and Computer Vision]: Miscellaneous

\section{General Terms}

Algorithms, Performance, Measurement, Experimentation

\section{Keywords}

Digital watermarking, Quality measurement, Ideal mapping curve, DWT, SPIHT.

Permission to make digital or hard copies of all or part of this work for personal or classroom use is granted without fee provided that copies are not made or distributed for profit or commercial advantage and that copies bear this notice and the full citation on the first page. To copy otherwise, to republish, to post on servers or to redistribute to lists, requires prior specific permission and/or a fee.

ICIMCS'11, August 5-7, 2011, Chengdu, Sichuan, China.

Copyright 2011 ACM 978-1-4503-0918-9/11/08 ...\$10.00.

\section{INTRODUCTION}

The evaluation of image quality is very important in today's video broadcasting, transmission control, and e-commerce, because quality is a key determinant of customer satisfaction and a key indicator of transmission condition [3]. Meanwhile, quality is very useful in the evaluation of the effectiveness or performance of image processing algorithms or systems. Generally speaking, the existing quality metrics can be classified into three categories based on whether the original image is available or not: the full-reference metrics, the partial reference metrics and the non-reference metrics. Due to the limited bandwidth of transmission channel, in some conditions, it is not practical to send the original signal to the receiver side, which makes the non-reference quality metric more desirable. The digital watermarking techniques can be used to design the non-reference quality measurement. In [3], a watermarking-based quality measurement is proposed to evaluate the quality change of an image against JPEG compression. Different textured images have different characteristics under the same distortion. The "Ideal Mapping Curve" is introduced as a reference curve to build the relationship between the degradation of the watermark and the degradation of the image. A binary watermark is embedded into the image. With the guidance of the "Ideal Mapping Curve", a feedback system is utilized to adjust the watermark embedding strength, according to how the JPEG compression affects the image quality, to enable the embedded watermark to reflect the image quality degradation. At the receiver side, the degraded watermark is extracted and the image quality is estimated in terms of some specific quality metric, e.g. PSNR, based on the degradation of the watermark. The advantage of the algorithm is the high accuracy it achieves in quality estimation. However, the feedback system used in the algorithm works in an iterative way that, to a certain degree, makes the algorithm time-consuming.

To improve the efficiency of the algorithm, we propose to embed watermark into an image with proper embedding strength decided by statistically analyzing the characteristics of the image against distortions. The bit-plane coding 


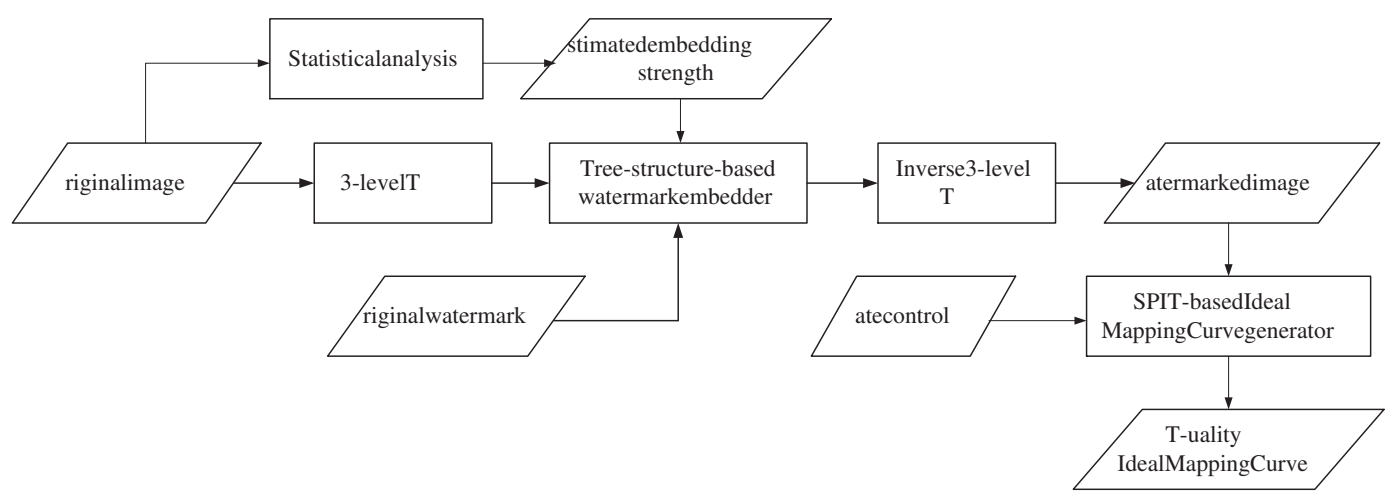

Figure 1: Watermark embedding and "Ideal Mapping Curve" generation.

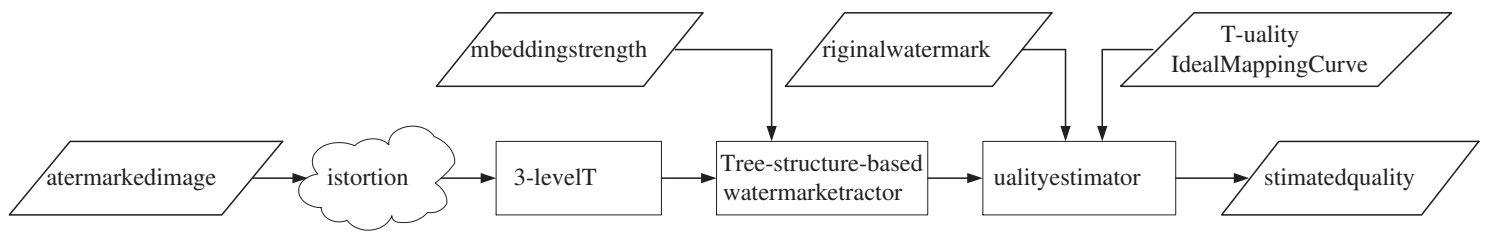

Figure 2: Watermark extraction and quality estimation.

mechanism is introduced into the watermark embedding, because based on the experiments, different bit-planes have different characteristics against distortions. However, for different distortions, the general rule is that with the increase of the distortion strength, the relatively lower bitplane will be affected more. The watermark is embedded into the DWT domain, so both the wavelet subbands and the wavelet coefficient bit-planes can be used to adjust the embedding strength. Basically, the watermark embedded in higher frequency wavelet subband and less significant bitplane will be more vulnerable to distortions and vice versa.

The concept of local watermark embedding presented in [4] helps theoretically analyze the image in details and provides effective guidance for the watermark embedding. In the meanwhile, similar to [3], an "Ideal Mapping Curve" is needed to define the relationship between the degradation of the watermark and the degradation of the host image. The SPIHT is a natural selection to localize the watermark embedding and summarize the "Ideal Mapping Curve" since the SPIHT works on the tree structure throughout the wavelet subbands and performs the bit-plane coding scan through the wavelet bit-planes [1][2]. Thus, the watermark embedding can be adjusted to accurately reflect how the SPIHT affects the image and watermark degradation under different BPP settings. This can make the watermark embedding adjustment faster and more accurate instead of relying on the iterative feedback process in [3] which works like a "black box". The "Ideal Mapping Curve" generated using the SPIHT works as a universal reference curve. For other distortions, such as JPEG compression and Gaussian noise pollution, the quality estimation can be performed by empirically weighting the universal "Ideal Mapping Curve".

The rest of the paper is organized as follows. Section 2 describes the proposed watermarking based image quality estimation algorithm. Section 3 discusses the implementation in details. Section 4 shows the experimental results. Section 5 concludes the paper.

\section{THE PROPOSED ALGORITHM}

To be able to estimate image quality, the watermark should be robust to distortions in some degree so that it still can be extracted even after the image is degraded. Moreover, the watermark should also be fragile so that it can reflect how much the image is degraded. Therefore, the watermark is desired to be semi-fragile. In this paper, the experiments will be focused on estimating image quality in terms of PSNR against JPEG compression and Gaussian noise pollution.

The watermark embedding and extraction schemes along with the "Ideal Mapping Curve" generation and quality estimation are shown in Fig. 1 and Fig. 2, respectively. As we can see in the figures, the watermark embedding and extraction schemes are implemented in the 3-level DWT domain to take advantage of both the spatial and the frequency information of the image. The tree structure based watermark embedder and extractor are developed based on the parentchildren relationship of the DWT coefficients expressed in [1][2]. A tree structure model generalized from [1] is illustrated in Fig. 3(a). All the coefficients categorized in the same color indicate a single tree. The number of trees for each image can be multiples of 3 . A 12-tree structure is shown in Fig. 3 (b). In the tree structure, a quarter of the DWT coefficients in the $L L$ block have no children. Among the rest of the coefficients, each has $4^{(4-\text { level })}$ children in one of the detailed DWT blocks in each level. By dividing the image into trees, the watermarking processes are localized. Theoretically, the finer the tree division, the bigger probability that the tree coefficients have similar characteristics, and the easier to control the watermark embedding strength. Based on the experiments, we divide the image into 6 trees. The watermark is evenly embedded into the DWT bit-planes of all the trees. Different bit-planes of the DWT coefficients have different robustness against certain distortion. A general rule is that the MSB plane is the most robust and the LSB plane is the least robust. To make it semi-fragile, we 
embed the watermark in the middle bit-planes. Another rule found in [3] is that the DWT coefficients in lower level is easier to be affected by distortions than those in higher level. Therefore, most of the watermark bits will be embedded into Level 2 and fewer into Level 3 and the least into Level 1. By observing the distribution of the DWT coefficients, the tree structure can also be used to adjust the locations for the watermark embedding to make the change of watermark TDR (True Detection Rate) consistent with the PSNR change of the host image.

After the watermark embedding, the inverse 3-level DWT is applied on the watermarked DWT coefficients to achieve the watermarked image. The SPIHT-based "Ideal Mapping Curve" generator is used to generate the universal TDRPSNR "Ideal Mapping Curve". The TDR of the extracted watermark defined in [3] will be calculated to evaluate the degradation of the watermark. The quality degradation of the image will be estimated by mapping the TDR to a quality value referring to the "Ideal Mapping Curve".

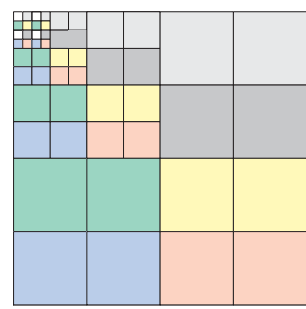

(a) Illustration of the tree categorization for watermarking.

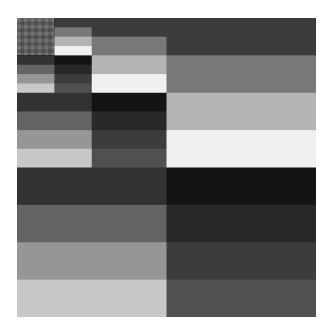

(b) The 12 trees generated on a $512 \times 512$ image.
Figure 3: The watermarking trees.

\section{IMPLEMENTATION STRATEGIES}

\section{1 "Ideal Mapping Curve" Generation}

The universal TDR-PSNR "Ideal Mapping Curve" generated using SPIHT coding is shown in Fig. 4. The BPP settings used in the curve generation is varying from 0.1 to 2 with a step of 0.1 to achieve the desired range for PSNR values. The curve is generated using 6 images with different types of characteristics, such as portrait, plants, animals, animation, scenery and building. For each image, a TDRPSNR curve is calculated using the SPIHT coding with the selected BPP settings. The universal "Ideal Mapping Curve" is generated by locally averaging the 6 calculated TDR-PSNR curves.

One more point needed to be noticed is that the tail of the universal "Ideal Mapping Curve" goes downwards straightly. The starting point of the vertical tail corresponds to a PSNR value of $32.5 \mathrm{~dB}$ and a TDR value of 0.3125 . This PSNR value is achieved when the BPP is very low which is less than 0.5. Under this situation, the image quality degrades severely and the watermark cannot be extracted. Thus, the image quality cannot be estimated accurately when the compression ratio is very high, i.e. the quality factor of the JPEG compression is 20 or lower or BPP is 0.5 or lower.

\subsection{Watermark Embedding and Extraction}

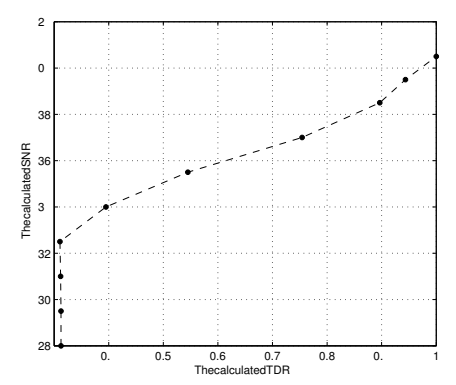

Figure 4: The universal "Ideal Mapping Curve".

To make the TDR change consistent with the PSNR change, the DWT coefficients for the watermark embedding are selected by observing the distribution of the $L L$ DWT coefficients of each tree. For a single tree, the corresponding coefficients in the $L L$ block are sorted in descent order and the average of the coefficients is denoted as $A v g$ with coordinate of $N_{A v g}$. The coefficients located between $\left[\beta\left(N_{A v g}-\frac{N_{w}}{2 N_{t}}\right)\right.$, $\left.\alpha\left(N_{A v g}+\frac{N_{w}}{2 N_{t}}\right)\right]$ are selected for the watermark embedding. The empirical value, $\alpha$, is bigger than 1 and $\beta$ is smaller than 1 so that the watermarked data will still be in the selected range and the embedded watermark bits can be located correctly. Here, we set $\alpha$ as 1.2 and $\beta$ as 0.8 .

For each selected DWT coefficient in the $L L$ block, we categorize it and all of its descents as a coefficient set for the watermark embedding. To increase the probability of the successful watermark extraction, we embed a watermark bit into 50 DWT coefficients of each selected coefficient set. Based on the experiments, the watermark will be embedded into the $4^{t h}$ bit-plane of the selected $L L$ coefficient and the $(l+1)^{t h}$ bit-plane of its descendants in the detail block in the $l^{\text {th }}$ DWT level.

If we denote the number of trees for a DWT image as $N_{t}$ and the number of the watermark bits as $N_{w}, N_{w} / N_{t}$ bits of watermark will be embedded into each DWT tree. To embed a watermark bit into a selected bit-plane of a specific coefficient, we need to check whether the value in the selected bit-plane is the same as the watermark bit. If they are the same, we do not need to change anything. Otherwise, we need to replace the value in the selected bit-plane with the value of the watermark bit.

In the watermark extraction scheme, the watermarked image goes through the JPEG compression and Gaussian noise pollution. The degraded watermarks are extracted and the TDRs are calculated. The quality of the degraded image is estimated by referring to the "Ideal Mapping Curve" of each distortion using linear interpolation.

\section{EXPERIMENTAL RESULTS}

The proposed scheme is tested under JPEG compression and Gaussian noise pollution. Ten different textured images are used in the experiments. The $32 \times 32$ binary watermark shown in [3] is used in the experiments. Two watermarked images are shown in Fig. 5 as examples. The PSNR's of the watermarked images are around $43 \mathrm{~dB}$.

Based on the experiments, the universal "Ideal Mapping Curve" can be directly used for the quality estimation against JPEG compression. The "Ideal Mapping Curve" for the Gaussian noise pollution can be achieved by weighting the 


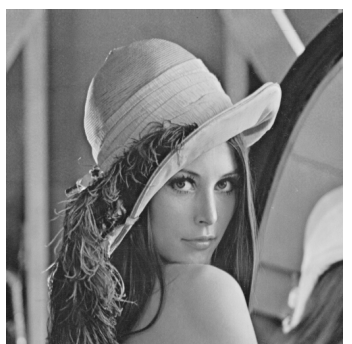

(a) $\mathrm{PSNR}=42.9851 \mathrm{~dB}$.

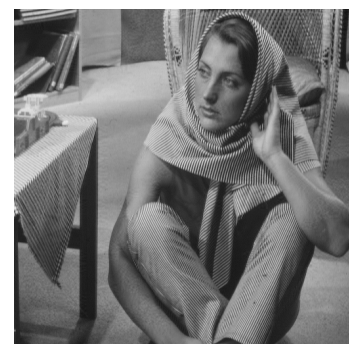

(b) $\mathrm{PSNR}=43.2001 \mathrm{~dB}$.
Figure 5: The watermarked images.

universal "Ideal Mapping Curve" with empirical factors of $\left[\begin{array}{llllllllll}1, & 0.9 & 0.9 & 0.9 & 0.9 & 0.9 & 0.9 & 0.9 & 0.9 & 0.9\end{array}\right]$.

For the experiments against JPEG compression, each image goes through JPEG compression with quality factors varying from 100 to 30 with a step of -10 . The compression with quality factors lower than 30 is not considered in this paper, because the image quality degraded too much under these compressions. After the quality degradation of the images, the TDR values are calculated. The quality of the degraded image is estimated by mapping the calculated TDR to a PSNR value referring to the "Ideal Mapping Curve". The PSNR values between the JPEG compressed images and the corresponding original images are calculated as a reference to evaluate the effectiveness of the proposed scheme.

The estimated quality values in terms of PSNR are shown in Fig. 6 with comparison to the calculated PSNR. The RMSE of the estimated quality values is $0.8788 \mathrm{~dB}$. The results are indicating that the proposed scheme is effective to estimate image quality in terms of PSNR against JPEG compression with very good accuracy.

Another set of experiments are implemented against Gaussian noise pollution. The results are shown in Fig. 7. The Gaussian noise is added to the watermarked image with variances varying from 0 to 20 with a step of 1 . The quality values of the distorted images are estimated in terms of PSNR. The calculated RMSE of the results in Fig. 7 is $0.5841 \mathrm{~dB}$. The experimental results show that the proposed scheme is also effective to estimate image quality against Gaussian noise pollution with high accuracy.

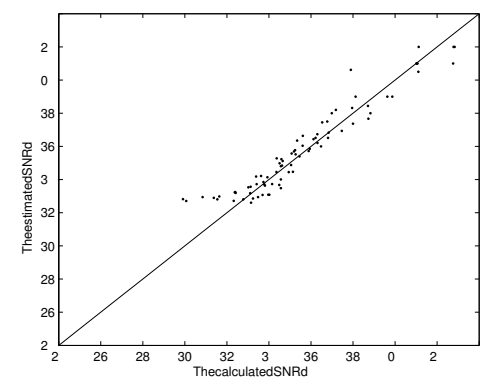

Figure 6: The experimental results tested on 10 different textured images against JPEG compression.

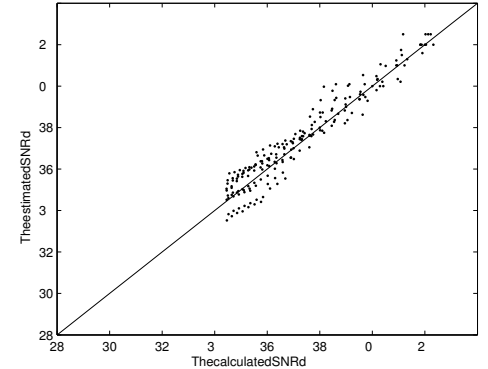

Figure 7: The experimental results tested on 10 different textured images against Gaussian noise pollution.

\section{CONCLUSIONS AND FUTURE WORK}

A tree-structured-watermarking-based image quality estimation scheme is proposed in the paper. The achievement needed to be mentioned is that the robustness of the watermark is decided by analyzing the image characteristics against the distortion and the feedback system in [3] is removed, which makes the proposed algorithm more efficient than the one presented in [3]. Another improvement is that the "Ideal Mapping Curve" used for image quality estimation against different distortions can be achieved by empirically weighting the universal "Ideal Mapping Curve". Based on the experiments, the proposed scheme is accurate for the image quality estimation against JPEG compression and Gaussian noise pollution.

More experiments are in progress. The universal "Ideal Mapping Curve" will be refined by using more different textured images. More training images will make the curve smoother, especially in the vertical tail. More test images will be used to evaluate the performance of the proposed scheme. The similar estimation accuracy can be expected. Moreover, more experiments will be conducted to demonstrate that the proposed quality estimation scheme works well against other distortions, such as low-pass filtering and packet-loss, in terms of other quality metrics, such as wPSNR, Watson JND and SSIM. We believe the proposed scheme will perform well as expected.

\section{REFERENCES}

[1] A. Said and W. Pearlman. A new, fast and efficient image codec based on set partitioning in hierachical trees. IEEE Transactions on Circuits and Systems for Video Technology, 6(3):243-250, June 1996.

[2] D. Taubma and M. Marcellin. JPEG2000: Image Compression Fundamentals, Standards and Practice. Kluwer Academic Publishers, ISBN: 0-7923-7519-X, 2004.

[3] S. Wang, D. Zheng, J. Zhao, W. J. Tam, and F. Speranza. An image quality evaluation method based on digital watermarking. IEEE Transactions on Circuits and Systems for Video Technology, 17(1):98-105, January 2007.

[4] D. Zheng, S. Wang, and J. Zhao. An RST invariant image watermarking algorithm with mathematical modeling and analysis of the watermarking processes. IEEE Transactions on Image Processing, 18(5):1055-1068, May 2009. 